\title{
Dialectical Logic and Boolean Algebra
}

\author{
Yaozhi Jiang \\ Correspondence: Yaozhi Jiang Shijiazhuang High-Tech District, Hebei, China.
}

Received: January 30, 2019 Accepted: March 11, 2019 Online Published: March 15, 2019

doi:10.5539/jmr.v11n2p92

URL: https://doi.org/10.5539/jmr.v11n2p92

\begin{abstract}
Dialectical logic was founded by German famous philosopher F. Hegel, but it has not been laid on mathematics for a long time. In this paper author explains the dialectical logic pure mathematically, and shows that the classic formal logic, its mathematical expression is Boolean algebra(includes multiple value system), is a special case from dialectical logic, and the true-valued function for dialectical logic is a continuous function valued on closed interval $[-1,+1]$ and defined on time-space axes system. The Aristotle three laws of formal logic are expanded into expression of dialectical logic, and Russell paradox is expanded into the case of multiple order. Some new theorems for Boolean operators and the matrix expression for De Morgan's theorem of multiple variables dialectical logic are given. At the end of the paper, linear or nonlinear dialectical logic are defined and analysis properties of dialectical logic true-valued function are pointed.
\end{abstract}

Keywords: dialectical logic, Boolean algebra, continuous true-valued function, Aristotle three laws, Russell paradox

\section{Introduction}

Dialectical logic was founded by German famous philosopher F. Hegel (Bencivenga,E, (2000));(Kosok,M. (1966));(Zong-kuan Zhao (2008)), but its mathematical expression has not been established for a long time. In this paper, as a successor of author's four papers published previously (Yaozhi Jiang. (2017)); (Yaozhi Jiang. (2018));(Yaozhi Jiang. (2018); Yaozhi Jiang. (2018)), author builds a pure mathematics for dialectical logic, and points out that relationship between "0-1" binary system Boolean algebra(includes multiple value system) and dialectical logic, formal logic which mathematical model is Boolean algebra(includes multiple true-valued function), is a special case of dialectical logic. But not similar with Boolean algebra(includes multiple value system), dialectical logic considers effects caused by the time, space and contradictions existed in the logical process. Dialectical logic does permit the state contradictions and does not permit the contradictions in causality law.

\section{Definitions and Symbols}

1.1. A dialectical logic space, denoted by $\mathfrak{R}=\left\{\Omega, \Omega^{*} ; f, f^{*}\right\}$,

In which research mapping $f: \Omega \rightarrow \Omega^{*}$, check mapping $f^{*}: \Omega^{*} \rightarrow \Omega$, where $\Omega$ is objective domain and $\Omega^{*}$ is subjective domain, the research mapping $f$ is a mapping that seek truth from objective domain $\Omega$ to subjective domain $\Omega^{*}$ and the checking mapping $f^{*}$ is a mapping that to check the truth sought from is "true or false" from the subjective domain $\Omega^{*}$ to the objective domain $\Omega$. But $f^{*} f \neq f f^{*}$, not satisfied by commutative law, and $f^{*} f=\nabla^{1} \neq e$, where $e$ is an unit mapping.

The objective domain $\Omega$ is called as real-source of subjective domain $\Omega^{*}$ upon researching mapping $f$ and checking mapping $f *$, and the subjective domain $\Omega^{*}$ is called as the mirror-image of objective domain $\Omega$ upon researching mapping $f$ and checking mapping $f^{*}$.

The objective domain $\Omega$ consists of TIME, SPACE, MATTER+ENERGY and OBJECTIVE INFORMATION, i.e. $\Omega=\{$ TIME, SPACE, MATTER + ENERGY, OBJECTIVE INFORMATION $\}$

And the subjective domain $\Omega^{*}=\{$ SUBJECTIVE INFORMATION $\}$

Actually we can treat with the objective domain $\Omega$ only by three-step: sensation, conceptualization and thinking in researching mapping $f$ and checking mapping $f^{*}$. With another word we can treat with only subjective information after sensation, i.e. $f:$ OBJECTIVE INFORMATION $\subset \Omega \rightarrow$ SUBJECTIVE INFORMATION $\subset \Omega^{*}$ or $f^{*}:$ SUBJECTIVE INFORMATION $\subset \Omega^{*} \rightarrow$ OBJECTIVE INFORMATION $\subset \Omega$.

Obviously the logic space $\mathfrak{R}$ is a complete space, i.e. for $\forall f, \exists f: \Omega \rightarrow \Omega^{*} \subset \Re$ and $\forall f^{*}$, 
$\exists f^{*}: \Omega^{*} \rightarrow \Omega \subset \mathfrak{R}$.

For $\forall \Omega, \exists f_{A} \subset f$, make $f_{A}: A \subset \Omega \rightarrow A^{*} \subset \Omega^{*}$

Then we call such logic space $\mathfrak{R}$ is recognizable.

And for $\forall A^{*} \subset \Omega^{*}, \exists f_{A}^{*} \subset f^{*}$ making

$f_{A}^{*}: A^{*} \subset \Omega^{*} \rightarrow A \subset \Omega$

we call such logic space $\mathfrak{R}$ is measurable.

For $\forall f, \exists f:\left(x_{0}, y_{0}, z_{0} ; t_{0}\right)$ and $\forall f^{*}, \exists f^{*}:\left(x_{0}, y_{0}, z_{0} ; t_{0}\right)$, make

$f:\left(x_{0}, y_{0}, z_{0} ; t_{0}\right) \rightarrow\left(x_{\infty}, y_{\infty}, z_{\infty} ; t_{\infty}\right)$ and $f^{*}:\left(x_{0}, y_{0}, z_{0} ; t_{0}\right) \rightarrow\left(x_{\infty}, y_{\infty}, z_{\infty} ; t_{\infty}\right)$.

Where $\left(x_{0}, y_{0}, z_{0} ; t_{0}\right)$ is the time-space point at present and $\left(x_{\infty}, y_{\infty}, z_{\infty} ; t_{\infty}\right)$ is the time-space point in the future till infinite future.

We call such logic space $\mathfrak{R}$ is predictable.

The logic spaces discussed by us all are recognizable, measurable, and predictable in this paper.

\subsection{Fact and Causality-Law}

While we say "this is a horse", it is the answer of problem" WHAT is this?", that is a fact can be sensed from objective domain, not that a causality can be obtained from subjective domain only by thinking and reasoning. Only when we explain the problems about the horse's states caused for WHY/HOW/WHAT/WHEN/WHERE, then the causality will be produced with. Fact is produced with conceptualization, and causality is produced with thinking and reasoning.

\subsection{True and False}

The problem about "True and False" of a logical proposition is an essential problem in all logic theory, and dialectical logic is not an exception with this point. In formal logic, its mathematical expression is Boolean algebra, the true value is a " $0-1$ " binary true-valued function, and in multiple value system the true value is multiple true-valued function. They have a common property that their true-valued function all are discrete function. Now in the dialectical logic, in briefly, we can call the dialectical logic is a logic whose true value is a continuous true-valued function valued on closed interval $[-1,+1]$ and defined on time-space axes system. There are broad differences between Boolean algebra(includes multiple value system) and dialectical logic, but they have a common mathematical gene: logic.

\subsection{Contradiction: State-Contradiction and Causality-Contradiction}

The contradiction is a most important topic in dialectical logic. In a logical system combined by several factors, these factors are struggling each other and depending on each other, their struggles are presented to antagonize each other or to be difference each other, and dependence among them are presented as "if you exist thus I must exist by you and struggle to you", and to be combined into one logic system. These factors which are struggling and depending on each other, we call them as contradiction in dialectical logic. This kind of contradiction above can be called as state-contradiction, and another kind of contradiction which produced by causality-law can be called as causality-contradiction. State-contradiction is either driving power of logic system or resistant power of logic system. With another word, we can say that the state-contradiction is a contradiction that these factors contradict each other in parallel and causality-contradiction is a contradiction that these factors contradict each other in serial.

The contradictions in parallel are contradictions in space and the contradictions in serial are contradictions in time.

In dialectical logic, we do permit the existence of state-contradiction and do not permit the existence of causality-contradiction.

If the causality-contradiction occurs, and if the causality-contradiction is in total factors then we call this kind causality-contradiction is all-wrong causality-contradiction, and if the causality-contradiction is in some factors not total factors then we call this kind causality-contradiction is partial-wrong causality-contradiction.

For the all-wrong causality-contradiction, this causality-law sought by us is senseless.For the partial-wrong causality-contradiction, this causality-law sought by us will be corrected or be improved.

\section{Some Axioms for Dialectical Logic}

Denote objective dialectical logic variable by $A^{ \pm n}=A^{ \pm n}(x, y, z ; t)$ and subjective dialectical logic variable by $A^{* \pm n}=A^{* \pm n}(x, y, z ; t)$, symbol" $\pm n "$ is that there are $n$ pairs of factors contradicting each other in the system, i.e. there are at most $\left(\begin{array}{l}2 n \\ 2\end{array}\right)=\frac{(2 n) !}{(2 n-2) !(2 n) !}$ state-contradictions in the dialectical logic variable $A^{ \pm n}$ or $A^{* \pm n}$, and 
$(x, y, z ; t)$ denotes the time-space axes acted on by the objective logic variable $A^{ \pm n}$ and subjective logic variable $A^{* \pm n}$. Sometimes we have

$\left\{\begin{array}{l}A^{ \pm n}(x, y, z ; t) \Leftrightarrow A^{ \pm n} \Leftrightarrow A \\ A^{* \pm n}(x, y, z ; t) \Leftrightarrow A^{* \pm n} \Leftrightarrow A^{*}\end{array}\right\}$ to brief the symbols.

Obviously we have below

$\Re=\left\{\Omega, \Omega^{*} ; f_{A} \subset f, f_{A}^{*} \subset f^{*}\right\}$,

$f_{A}: A^{ \pm n} \subset \Omega \rightarrow A^{* \pm n} \subset \Omega^{*}, f_{A}^{*}: A^{* \pm n} \subset \Omega^{*} \rightarrow A^{ \pm n} \subset \Omega$

If $\underbrace{f^{*} f f^{*} f \cdots f^{*} f}_{i-\text { pairs }}=\nabla^{i}$ then we call the $\nabla^{i}$ is the $i$-order circular mapping.

Axiom 1: Causality-law:

If logic space $\mathfrak{R}$ is recognizable, measurable, and predictable, $\forall\left\{A^{ \pm n} \in \Omega, A^{* \pm n} \in \Omega^{*}\right\} \subset \Re$, for a causality mapping $p$ and its inverse mapping $p^{-1}, p, p^{-1} \in f_{A}^{*}$,

$\exists p: C^{*} \subset A^{* \pm n} \rightarrow E^{*} \subset A^{* \pm n}$,

$\exists p^{-1}: E^{*} \subset A^{* \pm n} \rightarrow C^{*} \subset A^{* \pm n}, p^{-1} p=e, e$ is an unit mapping.

Where set $C^{*}$ is called as cause-set and set $E^{*}$ is called as effect-set.

If $\forall C^{*}=\varnothing$, then $\exists E^{*}=\varnothing$, and $\exists p=p^{-1}=0$.

By the way, the causality-law is repeatable to appear, if and only if the cause-set $C^{*} \neq \varnothing \Rightarrow E^{*} \neq \varnothing$ and causality mapping $p \neq 0 \Rightarrow p^{-1} \neq 0$. This is repeatablity of causality-law or call it as repeatablity condition of causality-law.

Axiom 2. The three-step, sensation, conceptualization and thinking, in researching mapping $f$ and checking mapping $f^{*}$, every one of them is a lossy information compression process.

Axiom 3: (Existence of error by circular mapping $\nabla^{i}$ )

For objective variable $A^{ \pm n} \subset \Omega$ and subjective variable $A^{* \pm n} \subset \Omega^{*}$, denote the error between $A^{* \pm n}$ and $A^{ \pm n}$ by $\nabla^{i}\left(A^{* \pm n}-A^{ \pm n}\right)$. We have: $\lim _{i \rightarrow \infty}\left(\nabla^{i}\left(A^{* \pm n}-A^{ \pm n}\right)\right)=0$, i.e. for every finite $i$, we have below $\varepsilon=\left(\nabla^{i}\left(A^{* \pm n}-A^{ \pm n}\right)\right)>0$

The Axiom 3. is produced by the Axiom 2. above, i.e. the errors were produced by the losses of information.

Axiom 4. The limiting process explained in Axiom 3. is an alternating convergence process, which is alternated by one-positive-after-one-negative.

Axiom 5. (Axiom for heredity and variation)

In an $i$ - order circular mapping $\nabla^{i}$, if exist a family of sets $\Phi_{i}=\left\{A_{i}^{* \pm n} ; \nabla^{i}: A_{i-1}^{* \pm n} \rightarrow A_{i}^{* \pm n}\right\}_{1}^{m}$, make $\bigcap_{1}^{m} \Phi_{i}=\bigcap_{1}^{m}\left\{A_{i}^{* \pm n}\right\}=A_{0} \neq \varnothing$, then we call $A_{0}$ as hereditary subset of family of sets $\Phi_{i}$ upon $i$ - order circular mapping $\nabla^{i}$, and the family of complementary sets $\bar{\Phi}_{i}=\left\{\widehat{A_{i}^{* \pm n}}-A_{0}\right\}$, where $\bar{\Phi}_{i}$ is the family of complementary sets of $\Phi_{i}$, then we call $\bar{\Phi}_{i}$ as family of variation sets of a family of sets $\Phi_{i}$.

For every circular mapping process in dialectical logic, there must exist heredity sets and variation sets.

Axiom 6. Any dialectical logic variable $A^{* \pm n}$ is infinitely fractal-able and finitely integral-able.

For dialectical logic variable $A^{* \pm n}$ with $n$ pairs of factors, in which every pair of factor can be fractal-ablized by a dialectical logic sub-variable $B^{* \pm n}$ into $A^{* B^{* \pm n}}$. In actual fact we can define a fractal-mapping $F$ make

$F\left(B^{* \pm n}\right): A^{* \pm n} \rightarrow A^{* B^{* \pm n}} \subset \Omega^{*}$

And an inverse mapping: integral-mapping $I$, make

$I\left(B^{* \pm n}\right): A^{* B^{* \pm n}} \rightarrow A^{* \pm n}$

Then we have below 


$$
\begin{aligned}
& F\left(B^{* \pm n}\right)_{1}^{\infty}: A^{* \pm n} \rightarrow A^{* B^{* \pm n}} \quad \text { and } \\
& I\left(B^{* \pm n}\right)_{1}^{j}: A^{* B^{* \pm n}} \rightarrow A^{* \pm n} \text {, for every finite } j .
\end{aligned}
$$

Where $F I=e, e$ is an unit mapping.

Axiom 7. Mozi principle(min-max principle)([3], Johnton, Ian(2010))

For every dialectical logic variable $A^{* \pm n}$, denote its gain-function by $G\left(A^{* \pm n}\right)$ and its cost-function by $C\left(A^{* \pm n}\right)$, we can explain the Mozi principle below: $\forall A^{* \pm n}$, this variant process must be satisfied with $\max \left(G\left(A^{* \pm n},\right), \min C\left(A^{* \pm n}\right)\right)$ or must be asymptotic convergence to $\max \left(G\left(A^{* \pm n},\right), \min C\left(A^{* \pm n}\right)\right)$, i.e. $\lim _{t \rightarrow \infty} \max \left(G\left(A^{* \pm n},\right), \lim _{t \rightarrow \infty} \min C\left(A^{* \pm n}\right)\right)$

Mozi principle produces a driving force to force the dialectical logic variable $A^{* \pm n}$ to choice the way for varying, this shows that the way for varying is complexity and sinuosity.

Axiom 8. (The energy conservation law of information)

The power function in information flow is every bit average information density $p(t)=\frac{-\log _{2} A(t)}{2^{n(t)}}$, where $A(t)=1-\left|\frac{a(t)-a^{\Uparrow}(t)}{a(t)}\right|$ that can be called as "non-fidelity of subjective information to objective information", obviously $0 \leq A(t) \leq 1 ; a^{\Uparrow}(t)$ is sampling function approaching to the function $y=a(t), n(t)$ is the bit number of sampling function. Flow function $f(t)$ in information is bit rate.

The power capacity function of information flow is: $P(t)=p(t) f(t)$.

The information meaning of power capacity function is information quantity flowed within unite time.

The work function of information flow is:

$$
W_{t_{0} \rightarrow t_{1}}(t)=\int_{t_{0}}^{t_{1}} P(t) d t=\int_{t_{0}}^{t_{1}} p(t) f(t) d t=\sum_{i=1}^{m} p\left(\Delta t_{i}\right) f\left(\Delta t_{i}\right) \Delta t_{i} ; \sum_{i=1}^{m} \Delta t_{i}=t_{1}-t_{0}
$$

The information meaning of work function is information quantity flowed within time interval $\left[t_{0}, t_{1}\right]$.

This axiom can be shown as $P\left(t_{1}\right)=p\left(t_{1}\right) f\left(t_{1}\right)=P\left(t_{2}\right)=p\left(t_{2}\right) f\left(t_{2}\right)$.

Axiom 9. Pareto principle (main factors principle)

$T\left(A^{* \pm n}\right)$ is mainly depended on a little number key factors. V. Pareto principle shows that we can hold these a little number main factors to obtain the main properties of $T\left(A^{* \pm n}\right)$.

\section{Boolean Operators Upon Dialectical Logic Variables and Some Lemmas}

For true-valued function $T\left(A^{* \pm n}(x, y, z ; t)\right) \in[-1,+1]$,

$$
T\left(A^{* \pm n}(x, y, z ; t)\right)\left\{\begin{array}{l}
>0 \\
=0 \\
<0
\end{array}\right.
$$

If $T\left(A^{* \pm n}(x, y, z ; t)\right)>0$, then we can call that the positive factors is superior than negative factors; if $T\left(A^{* \pm n}(x, y, z ; t)\right)=0$, then we can call that the positive factors is balance with the negative factors; if $T\left(A^{* \pm n}(x, y, z ; t)\right)<0$, then we can call that the negative factors is superior than the positive factors.

Obviously true-valued function $T\left(A^{* \pm n}(x, y, z ; t)\right) \in[-1,+1]$ is a continuous true value system for a dialectical logic proposition, i.e. the true-valued function explains the true-or-false for a dialectical logic proposition.

\subsection{Logic $\vee$ (Boolean Operator OR)}

For dialectical logic variables $A^{* \pm r}, A^{* \pm s}, A^{* \pm k}$, define logic $\vee$ below:

$$
T\left(A^{* \pm r}\right) \vee T\left(A^{* \pm s}\right)=\max \left\{T\left(A^{* \pm r}\right) ; T\left(A^{* \pm s}\right)\right\}
$$

The logic $\vee$ is satisfied by below

Commutative law: $T\left(A^{* \pm r}\right) \vee T\left(A^{* \pm s}\right)=T\left(A^{* \pm s}\right) \vee T\left(A^{* \pm r}\right)$

Associative law: $T\left(A^{* \pm r}\right) \vee\left(T\left(A^{* \pm s}\right) \vee T\left(A^{* \pm k}\right)\right)=\left(T\left(A^{* \pm r}\right) \vee T\left(A^{* \pm s}\right)\right) \vee T\left(A^{* \pm k}\right)$ 


\subsection{Logic $\wedge$ (Boolean AND)}

Define logic $\wedge$ below

$T\left(A^{* \pm r}\right) \wedge T\left(A^{* \pm s}\right)=\min \left\{T\left(A^{* \pm r}\right), T\left(A^{* \pm s}\right)\right\}$

Define logic $\wedge$ is satisfied by below

Commutative law: $T\left(A^{* \pm r}\right) \wedge T\left(A^{* \pm s}\right)=T\left(A^{* \pm s}\right) \wedge T\left(A^{* \pm r}\right)$

Associative law: $T\left(A^{* \pm r}\right) \wedge\left(T\left(A^{* \pm s}\right) \wedge T\left(A^{* \pm k}\right)\right)=\left(T\left(A^{* \pm r}\right) \wedge T\left(A^{* \pm s}\right)\right) \wedge T\left(A^{* \pm k}\right)$

3.3 Logic Hybrid Operating by Logic $\vee$ and Logic $\wedge$ (Hybrid Operating by Boolean OR and Boolean AND)

Logic hybrid arithmetic is satisfied by below

Distributive law: $T\left(A^{* \pm r}\right) \vee\left(T\left(A^{* \pm s}\right) \wedge T\left(A^{* \pm k}\right)\right)=\left(T\left(A^{* \pm r}\right) \vee T\left(A^{* \pm s}\right)\right) \wedge\left(T\left(A^{* \pm r}\right) \vee T\left(A^{* \pm k}\right)\right)$

$T\left(A^{* \pm r}\right) \wedge\left(T\left(A^{* \pm s}\right) \vee T\left(A^{* \pm k}\right)\right)=\left(T\left(A^{* \pm r}\right) \wedge T\left(A^{* \pm s}\right)\right) \vee\left(T\left(A^{* \pm r}\right) \wedge T\left(A^{* \pm k}\right)\right)$

3.4 Logic $\neg$ (Boolean NOT)

Define logic $\neg$ below

$$
\neg T\left(A^{* \pm r}\right)=\left\{\begin{array}{l}
+1-T\left(A^{* \pm r}\right), T\left(A^{* \pm r}\right)>0 \\
-1-T\left(A^{* \pm r}\right), T\left(A^{* \pm r}\right)<0 \\
0, T\left(A^{* \pm r}\right)=0
\end{array}\right.
$$

Denote $\underbrace{\neg \cdots \neg}_{p} T\left(A^{* \pm r}\right)=\neg^{p} T\left(A^{* \pm r}\right)$, then logic $\neg$ is satisfied by below

Idempotent law: $\underbrace{\neg \cdots \neg}_{p} T\left(A^{* \pm r}\right)=\left\{\begin{array}{l}T\left(A^{* \pm r}\right), p=2 m, m=1,2, \cdots \\ \neg T\left(A^{* \pm r}\right), p=2 m+1\end{array}\right.$

Mutual complementary law: $\neg T\left(A^{* \pm r}\right)+T\left(A^{* \pm r}\right)=\left\{\begin{array}{l}+1, T\left(A^{* \pm r}\right)>0 \\ -1, T\left(A^{* \pm r}\right)<0 \\ 0, T\left(A^{* \pm r}\right)=0\end{array}\right.$

\subsection{Lemma 1}

If two subsets $T^{+}, T^{-} \subset T\left(A^{* \pm n}\right)$ and $T^{+}>0, T^{-}<0$, then upon the operator logic $\neg$, there is inverse order law make

$$
T\left(A^{* \pm n}\right)=\left\{\begin{array}{l}
T_{a}^{+}>T_{b}^{+} \Rightarrow \neg\left(T_{a}^{+}>T_{b}^{+}\right)=\neg T_{a}^{+}(\neg>) \neg T_{b}^{+}=\neg T_{a}^{+}<\neg T_{b}^{+}, \\
T_{a}^{+}<T_{b}^{+} \Rightarrow \neg\left(T_{a}^{+}<T_{b}^{+}\right)=\neg T_{a}^{+}(\neg<) \neg T_{b}^{+}=\neg T_{a}^{+}>\neg T_{b}^{+}, T_{a}^{+}, T_{b}^{+} \in T^{+} ; \\
T_{a}^{-}>T_{b}^{-} \Rightarrow \neg\left(T_{a}^{-}>T_{b}^{-}\right)=\neg T_{a}^{-}(\neg>) \neg T_{b}^{-}=\neg T_{a}^{-}<\neg T_{b}^{-}, \\
T_{a}^{-}<T_{b}^{-} \Rightarrow \neg\left(T_{a}^{-}<T_{b}^{-}\right)=\neg T_{a}^{-}(\neg<) \neg T_{b}^{-}=\neg T_{a}^{-}>\neg T_{b}^{-}, T_{a}^{-}, T_{b}^{-} \in T^{-} ;
\end{array}\right.
$$

But

$$
T\left(A^{* \pm n}\right)=\left\{\begin{array}{l}
T^{+}>T^{-} \Rightarrow \neg\left(T^{+}>T^{-}\right)=\neg T^{+}(\neg>) \neg T^{-}=\neg T^{+}>\neg T^{-} \\
T^{-}<T^{+} \Rightarrow \neg\left(T^{-}<T^{+}\right)=\neg T^{-}(\neg<) \neg T^{+}=\neg T^{+}<\neg T^{+}
\end{array}\right.
$$

Actually we have shown that if operator $\neg \in T^{+}$only or $\neg \in T^{-}$only, then we obtain the result: $\neg>=<$ or $\neg<=>$, and if operator $\neg \in T^{+} \cup T^{-}$, then we obtain the result: $\neg>=>$ or $\neg<=<$.

The proof is obvious.

\subsection{Lemma 2}

If $T\left(A^{* \pm r}\right)>0$ and $T\left(A^{* \pm s}\right)>0$ or $T\left(A^{* \pm r}\right)<0$ and $T\left(A^{* \pm s}\right)<0$ or $T\left(A^{* \pm r}\right)=T\left(A^{* \pm s}\right)=0$, operator $\vee$ and $\wedge$ is a pair of reciprocal operator,

and $\wedge \vee=\vee \wedge=1, \neg \wedge=\vee, \neg \vee=\wedge$.

Proof: $\neg(\vee)=-(\max )=\min =\wedge$ and $\neg(\wedge)=-(\min )=\max =\vee$. 


$$
\wedge \vee\left(T\left(A^{* \pm r}\right), T\left(A^{* \pm s}\right)\right)=\left\{\begin{array}{l}
\max \left(T\left(A^{* \pm r}\right), T\left(A^{* \pm s}\right)\right) \\
\min \left(T\left(A^{* \pm r}\right), T\left(A^{* \pm s}\right)\right)
\end{array}\right\}=\left(T\left(A^{* \pm r}\right), T\left(A^{* \pm s}\right)\right)
$$

Therefor they are reciprocal operator, thus $\wedge \vee=\vee \wedge=1$. For $\neg \wedge=\vee, \neg \vee=\wedge$, they are obvious.

Proof is over.

\subsection{Lemma 3}

If $T\left(A^{* \pm r}\right)>0$ and $T\left(A^{* \pm s}\right)<0$ or $T\left(A^{* \pm r}\right)<0$ and $T\left(A^{* \pm s}\right)>0$, operator $\vee$ and $\wedge$ is satisfied by $\wedge \vee=\vee \wedge=1$, $\neg \wedge=\wedge, \neg \vee=\vee$.

$$
\begin{aligned}
& \text { Proof: } \hat{\wedge} \vee=\vee \wedge=1 \text { is } \quad \text { obvious } \quad \text { as } \quad \text { in } \quad \text { Lemma } \quad \mathbf{2 .} \text { above, } \quad \text { and } \quad \text { if } \quad T\left(A^{* \pm r}\right)>0 \quad, \\
& \neg \wedge=\neg(\min )=\left(-1-\left(T\left(A^{* \pm s}\right)<0\right)\right)<0=\min =\wedge \\
& \neg \vee=\neg(\max )=\left(+1-T\left(A^{* \pm r}\right)>0\right)>0=\max =\vee
\end{aligned}
$$

With same way for the case of $T\left(A^{* \pm r}\right)<0$ and $T\left(A^{* \pm s}\right)>0$, we can obtain the result as same as above.

These are because of the definitions about complementary operator make the negative operated into negative and the positive operated into positive, but the positive is larger than the negative forever.

Proof is over.

\subsection{Lemma 4}

(Distributed law for operator $\neg$ one-by-one towards right)

If $T\left(A^{* \pm r}\right)>0$ and $T\left(A^{* \pm s}\right)>0$ or $T\left(A^{* \pm r}\right)<0$ and $T\left(A^{* \pm s}\right)<0$ or $T\left(A^{* \pm r}\right)=T\left(A^{* \pm s}\right)=0$ :

$\neg\left(T\left(A_{i}\right) \vee T\left(A_{j}\right)\right)=\neg T\left(A_{i}\right)(\neg(\vee)) \neg T\left(A_{j}\right)=\neg T\left(A_{i}\right) \wedge \neg T\left(A_{j}\right)$

$\neg\left(T\left(A_{i}\right) \wedge T\left(A_{j}\right)\right)=\neg T\left(A_{i}\right)(\neg \wedge) \neg T\left(A_{j}\right)=\neg T\left(A_{i}\right) \vee \neg T\left(A_{j}\right)$

This is just De Morgan's theorem below for the cases of $T\left(A^{* \pm r}\right)>0$ and $T\left(A^{* \pm s}\right)>0$ or $T\left(A^{* \pm r}\right)<0$ and $T\left(A^{* \pm s}\right)<0$ or $T\left(A^{* \pm r}\right)=T\left(A^{* \pm s}\right)=0$.

Proof: Use De Morgan's theorem shown below can obtain the result easily.

\subsection{Lemma 5}

Operator $\neg$ is reciprocal operator to itself, i.e. $\neg \neg=1$.

Proof: obviously based on the Idempotent law in section 3.4.

3.10 Lemma 6

Suppose operator mapping $F$ and set $S, S_{1}$, and $S_{1} \subset S$, if

$F: S \rightarrow S$ and $F: S_{1} \rightarrow S_{1}$, then we called operator mapping $F$ is self-completed separately for sets $S$ and $S_{1}$, and denoted by $F:\left\{S \supset S_{1}\right\} \mapsto$ s.c. If we know

$F_{k}:\left\{S \supset S_{i}\right\} \mapsto s . c, i$ and $k$ is independent natural number, and

$F_{k}: S \rightarrow S$, then we have $F_{k}: S_{1} \rightarrow S_{1}$.

3.11 Three Laws of Aristotle (A.C. 384-A.C.322) and Russell Paradox

Now we explain the Aristotle three law in formal logic as expanded expression in dialectical logic, note that these laws only for case of causality-contradiction.

3.11.1 Law of Identity(Reflexive Law)

$T\left(A^{* \pm n}(x, y, z ; t)\right)=T\left(A^{* \pm n}(x, y, z ; t)\right)$

3.11.2 Law of Contradiction

Denote cause set by $C=\left\{c_{1}, c_{2}, \cdots, c_{m}\right\}$, effect set by $E=\left\{e_{1}, e_{2}, \cdots, e_{k}\right\}$, and cause-effect mapping by $p_{1}$, if dialectical logic proposition

$p_{1}: C \rightarrow E$

is true, then anyone proposition of below

$\neg p_{1}: C \rightarrow E ; \neg p_{1}: \neg C \rightarrow E ; \neg p_{1}: \neg C \rightarrow \neg E ; p_{1}: \neg C \rightarrow E ; p_{1}: \neg C \rightarrow \neg E ;$

$p_{1}: C \rightarrow \neg E ; \neg p_{1}: C \rightarrow \neg E ;$ 
is not also true with $p_{1}: C \rightarrow E$ at same time.

\subsubsection{Law of Excluded Middle}

If dialectical logic propositions below $\neg p_{1}: C \rightarrow E ; \neg p_{1}: \neg C \rightarrow E ; \neg p_{1}: \neg C \rightarrow \neg E ; p_{1}: \neg C \rightarrow E ; p_{1}: \neg C \rightarrow \neg E$;

$p_{1}: C \rightarrow \neg E ; \neg p_{1}: C \rightarrow \neg E$;

all are false, then $p_{1}: C \rightarrow E$ is not also false with anyone proposition of above at same time.

3.11.4 Russell Paradox

A famous example of formal logic paradox is the Russell paradox( by B.A.W. Russell, 1901)

Denote set $A=\left\{a_{i}, i=1,2, \cdots, n\right\}$, and logic mappings $f_{1}, f_{2}, \cdots, f_{s}$, make

$f_{1}(A) \rightarrow A_{1} \subset A$,

$f_{2}(A) \rightarrow A_{2} \subset A$,

$\cdots$

$f_{s}(A) \rightarrow A_{s} \subset A$.

If $\forall A_{j}, A_{k}, k \neq j$ and $j, k \leq s, \exists f_{j}, f_{k}$ is contradicted each other in meaning of formal logic and $A_{j} \cap A_{k}=A_{j, k} \neq \varnothing$, then we call below

$F_{j, k}=\left(f_{k}, f_{j}, A_{j, k}\right)$

as a binary Russell paradox.

A Chinese ancient example of Russell paradox is the idiom: self-contradictory.

If $\underbrace{\forall A_{j}, \cdots, A_{k}}_{r}$, and $j \neq k, \cdots, j, \cdots, k \leq s, \exists f_{j}, \cdots, f_{k}$ is contradicted to any other in meaning of formal logic and

$$
\bigcap_{\text {every:j, }, \cdots, k} A_{j, \cdots, k}=A_{j, \cdots, k} \neq \varnothing
$$

Then we call below

$$
F_{j, \cdots, k}=\left(f_{k}, \cdots, f_{j}, A_{j, \cdots, k}\right)
$$

as a $r$-order Russell paradox.

Theorem 3.1. The condition to avoid Russell paradox is that below: a, logic mappings are not contradicted in meaning of formal logic; $\mathrm{b}, A_{j, \ldots, k}=\varnothing$, is satisfied the one of two cases at least.

This theorem's proof is obviously by the definition.

\section{Some Theorems}

\subsection{Theorem 1. (Magnitude Theorem)}

If $T\left(A^{* \pm n}\right) \in[-1,+1]$ is satisfied by the regulations shown in section 3., then $\alpha T\left(A^{* \pm n}\right) \in[-\alpha,+\alpha]$ is also satisfied by the regulations shown in section 3., where $\alpha>0$ is a positive constant.

Proof: After timed by $\alpha, \alpha T\left(A^{* \pm n}\right) \in[-\alpha,+\alpha]$ is also satisfied by all of the regulations shown in section 3., this can be checked one-by-one by regulations shown in section 3, therefor $\alpha T\left(A^{* \pm n}\right) \in[-\alpha,+\alpha]$ is also satisfied by the regulations shown in section 3 .

\section{Proof is over.}

\subsection{Theorem 2. (Phase Theorem)}

If $\beta \geq \alpha$, where $\beta$ is a positive constant, then $\left(\beta+\alpha T\left(A^{* \pm n}\right)\right)=T\left(A_{\alpha, \beta}^{* \pm n}\right) \in[\beta-\alpha, \beta+\alpha]$ is also satisfied by all of the regulation shown in section 3 ..

Proof: Because of the closed interval is $[\beta-\alpha \geq 0, \beta+\alpha]$, thus the true-valued function is not any negative number and avoid the negative true value is also operated into negative number upon operator logic $\neg$.

Proof is over.

\subsection{Theorem 3}

Boolean algebra in " $0-1$ " binary system and multiple value system is the special case of dialectical logic, the case is without consideration for existence of time $t$, space $(x, y, z)$ and state-contradictions. 
Proof: If without consideration for time $t$ and space $(x, y, z)$, then the true-valued function of Boolean algebra is valued on two discrete value of $S=\{0,1\} \in[0,+1]$, and multiple true-valued function is valued on several discrete value of $X=\left\{a_{1}, a_{2}, \cdots, a_{i}\right\} \in[0,+1]$, and under this condition the true-valued function of dialectical logic is a continuous function valued on closed interval $D=[-1,+1]$, then we have below: $S \subset D, X \subset D$; and $S$ or $X$ separately self-completed for operators of $\wedge, \vee, \neg$, thus we know that: suppose $S \subset D, X \subset D$; and $S$ or $X$ separately self-completed for operators of $\wedge, \vee, \neg$, Based on Lemma 6. if dialectical logic true-valued function satisfies the operation law above, then binary Boolean algebra true-valued function and multiple true-valued function must satisfy the operation laws above.

Proof is over.

\section{Theorem 4. De Morgan's Theorem}

Case 1. If $T\left(A^{* \pm r}\right)>0$ and $T\left(A^{* \pm s}\right)>0$ or $T\left(A^{* \pm r}\right)<0$ and $T\left(A^{* \pm s}\right)<0$ or $T\left(A^{* \pm r}\right)=T\left(A^{* \pm s}\right)=0$ :

$\neg\left(T\left(A^{* \pm r}\right) \wedge T\left(A^{* \pm s}\right)\right)=\left(\neg T\left(A^{* \pm r}\right)\right) \vee\left(\neg T\left(A^{* \pm s}\right)\right)$

$\neg\left(T\left(A^{* \pm r}\right) \vee T\left(A^{* \pm s}\right)\right)=\left(\neg T\left(A^{* \pm r}\right)\right) \wedge\left(\neg T\left(A^{* \pm s}\right)\right)$

Case 2. If $T\left(A^{* \pm r}\right)>0$ and $T\left(A^{* \pm s}\right)<0$ or $T\left(A^{* \pm r}\right)<0$ and $T\left(A^{* \pm s}\right)>0$ :

$\neg\left(T\left(A^{* \pm r}\right) \wedge T\left(A^{* \pm s}\right)\right)=\left(\neg T\left(A^{* \pm r}\right)\right) \wedge\left(\neg T\left(A^{* \pm s}\right)\right)$

$\neg\left(T\left(A^{* \pm r}\right) \vee T\left(A^{* \pm s}\right)\right)=\left(\neg T\left(A^{* \pm r}\right)\right) \vee\left(\neg T\left(A^{* \pm s}\right)\right)$

The proof is so obvious that to be unnecessary.

Theorem 5. De Morgan's theorem in multiple-variable dialectical logic by matrices:

Denote the dialectical logic variable set by $A=\left\{A_{1}, A_{2}, \cdots, A_{j}\right\}$, then define matrices

$$
\begin{aligned}
T(A) & =\left[\begin{array}{cccc}
T\left(A_{1}\right) \vee T\left(A_{1}\right) & T\left(A_{1}\right) \vee T\left(A_{2}\right) & \cdots & T\left(A_{1}\right) \vee T\left(A_{j}\right) \\
T\left(A_{2}\right) \vee T\left(A_{1}\right) & T\left(A_{2}\right) \vee T\left(A_{2}\right) & \cdots & T\left(A_{2}\right) \vee T\left(A_{j}\right) \\
\cdots & \cdots & \ddots & \ldots \\
T\left(A_{j}\right) \vee T\left(A_{1}\right) & T\left(A_{j}\right) \vee T\left(A_{2}\right) & \cdots & T\left(A_{j}\right) \vee T\left(A_{j}\right)
\end{array}\right] \\
T \hat{(A)} & =\left[\begin{array}{cccc}
T\left(A_{1}\right) \wedge T\left(A_{1}\right) & T\left(A_{1}\right) \wedge T\left(A_{2}\right) & \cdots & T\left(A_{1}\right) \wedge T\left(A_{j}\right) \\
T\left(A_{2}\right) \wedge T\left(A_{1}\right) & T\left(A_{2}\right) \wedge T\left(A_{2}\right) & \cdots & T\left(A_{2}\right) \wedge T\left(A_{j}\right) \\
\cdots & \cdots & \ddots & \cdots \\
T\left(A_{j}\right) \wedge T\left(A_{1}\right) & T\left(A_{j}\right) \wedge T\left(A_{2}\right) & \cdots & T\left(A_{j}\right) \wedge T\left(A_{j}\right)
\end{array}\right]
\end{aligned}
$$

Case 1. If $T\left(A^{* \pm r}\right)>0$ and $T\left(A^{* \pm s}\right)>0$ or $T\left(A^{* \pm r}\right)<0$ and $T\left(A^{* \pm s}\right)<0$ or $T\left(A^{* \pm r}\right)=T\left(A^{* \pm s}\right)=0$ :

$$
\begin{gathered}
\neg T(A)=\left[\begin{array}{cccc}
\neg T\left(A_{1}\right) \wedge \neg T\left(A_{1}\right) & \neg T\left(A_{1}\right) \wedge \neg T\left(A_{2}\right) & \cdots & \neg T\left(A_{1}\right) \wedge \neg T\left(A_{j}\right) \\
\neg T\left(A_{2}\right) \wedge \neg T\left(A_{1}\right) & \neg T\left(A_{2}\right) \wedge \neg T\left(A_{2}\right) & \cdots & \neg T\left(A_{2}\right) \wedge \neg T\left(A_{j}\right) \\
\cdots & \cdots & \ddots & \ldots \\
\neg T\left(A_{j}\right) \wedge \neg T\left(A_{1}\right) & \neg T\left(A_{j}\right) \wedge \neg T\left(A_{2}\right) & \cdots & \neg T\left(A_{j}\right) \wedge \neg T\left(A_{j}\right)
\end{array}\right] \\
\neg \hat{T(A)}=\left[\begin{array}{cccc}
\neg T\left(A_{1}\right) \vee \neg T\left(A_{1}\right) & \neg T\left(A_{1}\right) \vee \neg T\left(A_{2}\right) & \cdots & \neg T\left(A_{1}\right) \vee \neg T\left(A_{j}\right) \\
\neg T\left(A_{2}\right) \vee \neg T\left(A_{1}\right) & \neg T\left(A_{2}\right) \vee \neg T\left(A_{2}\right) & \cdots & \neg T\left(A_{2}\right) \vee \neg T\left(A_{j}\right) \\
\cdots & \ldots & \ddots & \ldots \\
\neg T\left(A_{j}\right) \vee \neg T\left(A_{1}\right) & \neg T\left(A_{j}\right) \vee \neg T\left(A_{2}\right) & \cdots & \neg T\left(A_{j}\right) \vee \neg T\left(A_{j}\right)
\end{array}\right]
\end{gathered}
$$

Case 2. If $T\left(A^{* \pm r}\right)>0$ and $T\left(A^{* \pm s}\right)<0$ or $T\left(A^{* \pm r}\right)<0$ and $T\left(A^{* \pm s}\right)>0$ :

$$
\neg T(A)=\left[\begin{array}{cccc}
\neg T\left(A_{1}\right) \vee \neg T\left(A_{1}\right) & \neg T\left(A_{1}\right) \vee \neg T\left(A_{2}\right) & \cdots & \neg T\left(A_{1}\right) \vee \neg T\left(A_{j}\right) \\
\neg T\left(A_{2}\right) \vee \neg T\left(A_{1}\right) & \neg T\left(A_{2}\right) \vee \neg T\left(A_{2}\right) & \cdots & \neg T\left(A_{2}\right) \vee \neg T\left(A_{j}\right) \\
\ldots & \ldots & \ddots & \ldots \\
\neg T\left(A_{j}\right) \vee \neg T\left(A_{1}\right) & \neg T\left(A_{j}\right) \vee \neg T\left(A_{2}\right) & \cdots & \neg T\left(A_{j}\right) \vee \neg T\left(A_{j}\right)
\end{array}\right]
$$




$$
\neg \hat{T(A)}=\left[\begin{array}{cccc}
\neg T\left(A_{1}\right) \wedge \neg T\left(A_{1}\right) & \neg T\left(A_{1}\right) \wedge \neg T\left(A_{2}\right) & \cdots & \neg T\left(A_{1}\right) \wedge \neg T\left(A_{j}\right) \\
\neg T\left(A_{2}\right) \wedge \neg T\left(A_{1}\right) & \neg T\left(A_{2}\right) \wedge \neg T\left(A_{2}\right) & \cdots & \neg T\left(A_{2}\right) \wedge \neg T\left(A_{j}\right) \\
\cdots & \ldots & \ddots & \ldots \\
\neg T\left(A_{j}\right) \wedge \neg T\left(A_{1}\right) & \neg T\left(A_{j}\right) \wedge \neg T\left(A_{2}\right) & \cdots & \neg T\left(A_{j}\right) \wedge \neg T\left(A_{j}\right)
\end{array}\right]
$$

These matrices are the De Morgan's theorem in matrix expression for multiple variable dialectical logic. These matrices all are symmetrical with main diagonal line.

Its proof is obvious.

\section{Linear Dialectical Logic and Nonlinear Dialectical Logic}

For causality mapping $p_{1} \in p$ and their inverse mapping

$$
\begin{aligned}
& p_{1}^{-1} \in p^{-1}, p_{1} p_{1}^{-1}=p p^{-1}=1, \text { and cause-set } C_{1} \subset C, \text { effect-set } E_{1} \subset E, \\
& \forall p_{1}: C_{1} \rightarrow E_{1},
\end{aligned}
$$

Case1.

$$
\begin{aligned}
& \exists p_{1}:\left(C_{1}=C_{1,1} \cup C_{1,2} \neq \varnothing\right) \rightarrow E_{1}=E_{1,1} \cup E_{1,2} \Rightarrow \\
& \Rightarrow\left(p_{1}: C_{1,1}\right) \cup\left(p_{1}: C_{1,2}\right) \rightarrow E_{1,1} \cup E_{1,2} \neq \varnothing \Rightarrow \\
& \Rightarrow\left(p_{1}: C_{1,1} \rightarrow E_{1,1}\right) \cup\left(p_{1}: C_{1,2} \rightarrow E_{1,2}\right)=E_{1,1} \cup E_{1,2} \neq \varnothing
\end{aligned}
$$

We can call the causality-law is union-set-linear.

Case2.

$$
\begin{aligned}
& \exists p_{1}:\left(C_{1}=C_{1,1} \cap C_{1,2} \neq \varnothing\right) \rightarrow E_{1}=E_{1,1} \cap E_{1,2} \Rightarrow \\
& \Rightarrow\left(p_{1}: C_{1,1}\right) \cap\left(p_{1}: C_{1,2}\right) \rightarrow E_{1,1} \cap E_{1,2} \neq \varnothing
\end{aligned}
$$

We can call the causality-law is intersection-set-linear.

If the causality-law is both of union-set-linear and intersection-set-linear, then we call the causality-law is pure-linear. And otherwise are called as nonlinear.

Linear or nonlinear causality-law is also can be called as linear dialectical logic or nonlinear dialectical logic.

\subsection{Theorem 6}

If a causality-law is only union-set-linear, then we have either of below:

Case1.

$$
\begin{aligned}
& p_{1}:\left(C_{1}=C_{1,1} \cap C_{1,2} \neq \varnothing\right) \rightarrow E_{1} \supset E_{1,1} \cap E_{1,2} \Rightarrow \\
& \Rightarrow\left(p_{1}: C_{1,1}\right) \cap\left(p_{1}: C_{1,2}\right) \rightarrow E_{1,1} \cap E_{1,2} \neq \varnothing
\end{aligned}
$$

or Case 2.

$$
\begin{aligned}
& p_{1}:\left(C_{1}=C_{1,1} \cap C_{1,2} \neq \varnothing\right) \rightarrow E_{1}=E_{1,1} \cap E_{1,2} \Rightarrow \\
& \Rightarrow\left(p_{1}: C_{1,1}\right) \cap\left(p_{1}: C_{1,2}\right) \rightarrow E_{1,1} \cap E_{1,2} \neq \varnothing
\end{aligned}
$$

Proof: it's obvious.

Poof is over.

\subsection{Lemma 6}

If $E_{1}=E_{1,1} \cup E_{1,2}$, then $E_{1}=E_{1,1} \cap E_{1,2} \Rightarrow E_{1}=E_{1,1}=E_{1,2}$.

Proof: It's obvious. 
Proof is over.

5.3 Theorem 7. From Lemma 6

for a pure-linear causality-law we have below:

$p_{1}:\left(C_{1}=C_{1,1} \cap C_{1,2} \neq \varnothing\right) \rightarrow E_{1}=E_{1,1} \cap E_{1,2} \Rightarrow$

$\Rightarrow\left(p_{1}: C_{1,1}\right) \cap\left(p_{1}: C_{1,2}\right) \rightarrow E_{1}=E_{1,1}=E_{1,2} \neq \varnothing$

Proof: From Lemma 6., it's obvious.

Proof is over.

So we can sure that the union-set-linear causality-law is divided into two cases below:

Case 1.: if $E_{1}=E_{1,1} \cup E_{1,2}$, then $E_{1}=E_{1,1} \cap E_{1,2} \Rightarrow E_{1}=E_{1,1}=E_{1,2}$;

Case 2.: if $E_{1}=E_{1,1} \cup E_{1,2}$, then $E_{1} \supset E_{1,1} \cap E_{1,2} \Rightarrow E_{1} \neq E_{1,1} \cap E_{1,2}$.

The cases of nonlinear causality-law is complexity and in huge number of.

\section{Some Properties in Analysis of True-Valued Function $T\left(A^{* \pm n}\right)$}

For continuous true-valued function and any-order partial derived number are all existent, the differentiation of true-valued function $T\left(A^{* \pm n}\right) \in[-1,+1], A^{* \pm n}=A^{* \pm n}(x, y, z ; t)$, have some properties below:

6.1 Total Differential Equation

$$
\begin{aligned}
& d T=\frac{\partial T}{\partial x} d x+\frac{\partial T}{\partial y} d y+\frac{\partial T}{\partial z} d z+\frac{\partial T}{\partial t} d t, T=T\left(A^{* \pm n}(x, y, z ; t)\right) \\
& d^{2} T=\frac{\partial^{2} T}{\partial x^{2}} d x^{2}+\frac{\partial^{2} T}{\partial y^{2}} d y^{2}+\frac{\partial^{2} T}{\partial z^{2}} d z^{2}+\frac{\partial^{2} T}{\partial t^{2}} d t^{2}+ \\
& +2 \frac{\partial^{2} T}{\partial x \partial y} d x d y+2 \frac{\partial^{2} T}{\partial x \partial z} d x d z+2 \frac{\partial^{2} T}{\partial x \partial t} d x d t+2 \frac{\partial^{2} T}{\partial y \partial z} d y d z+2 \frac{\partial^{2} T}{\partial y \partial t} d y d t+2 \frac{\partial^{2} T}{\partial z \partial t} d z d t
\end{aligned}
$$

From above we can solute the problems of mini-max value in true-valued function.

\subsection{Average Value of True-Valued Function}

Denote the average value of true-valued function $T\left(A^{* \pm n}(x, y, z ; t)\right)$ by

$$
\begin{gathered}
\bar{T}=\sum_{k=1}^{m} \frac{\iiint_{I_{k} \in T>0}\left(T\left(A^{* \pm n}(x, y, z ; t)\right)>0\right) d x d y d z d t}{\left(\Delta x_{k} \Delta y_{k} \Delta z_{k} \Delta t_{k}\right)}+ \\
+\sum_{m=1}^{p} \frac{\iiint_{I_{m} \in T<0} \int\left(T\left(A^{* \pm n}(x, y, z ; t)\right)<0\right) d x d y d z d t}{\left(\Delta x_{m} \Delta y_{m} \Delta z_{m} \Delta t_{m}\right)}
\end{gathered}
$$

Where $I_{k} \in T>0$ is the all areas that true-valued function is larger than zero and $I_{m} \in T<0$ is the all areas that true-valued function is smaller than zero.

This average value of true-valued function can be used for the whole properties of true-valued function in dialectical logic.

\section{References}

Bencivenga, E. (2000). Hegel's Dialectical logic, Oxford University Press.

Johnston, I. (2010). The Mozi: A complete Translation, Hongkong: The Chinese University Press.

Kosok, M. (1966). The formalization of Hegel's Dialectical logic, International Philosophical Quarterly, IPQ6(4), 596-631, January. https://doi.org/10.5840/jpq 19666456

Yaozhi, J. (2017). Dialectical Logic K-Modle: A Mathematical Model for Machine, Journal of Mathematics Research, 
6(9). Canadian Center of Science and Education. https://doi.org/10.5539/jmr.v9n6p82

Yaozhi, J. (2018). Dialectical K-Model: The Discrete Time Dynamical Sampling System, Multidimensional Logic Variable and Associate Database(ABD), Journal of Mathematics Research, 2(10). Canadian Center of Science and Education. lhttps://doi.org/10.5539/jmr.v10n2p88

Yaozhi, J. (2018). Dialectical Logic K-Model: On Multidimensional Discrte Dynamical Sampling System and Further Properties of Kirchhoff Matrices, Journal of Mathematics Research, 3(10). Canadian Center of Science and Education. https://doi.org/10.5539/jmr.v10n3p20

Yaozhi, J. (2018). Dialectical logic K-Model:Some Applications by Fuzzy-Probability Theory, Cause-Effect Analysis, Chaos Dynamics and Optimization Theory, Transactions on Machine Learning and Artificial Intelligence, 6(6), 2018, Society for Science and Education United Kingdom. http://dx.doi.org/10.14738/tmlai.66.5862

Zong-kuan, Z. (2008). From Classical logic to Mathematical Dialectical logic, Journal of Bijie University, 2008-01(in Chinese).

\section{Copyrights}

Copyright for this article is retained by the author(s), with first publication rights granted to the journal.

This is an open-access article distributed under the terms and conditions of the Creative Commons Attribution license (http://creativecommons.org/licenses/by/4.0/). 